\title{
Extramammary Paget disease of the glans penis
}

\author{
Yue-Tong Qian MD, Dong-Lai Ma MD PhD \\ Cite as: CMAJ 2018 September 24;190:E1142. doi: 10.1503/cmaj.180348
}

A 63-year-old man presented to the dermatology clinic with a three-year history of an erythematous patch with occasional pruritis that covered his glans penis and urethral meatus. Physical examination showed an irregular erythematous plaque that was circumscribed and infiltrated (Figure 1). No inguinal lymphadenopathy was found. The patient's medical history included a low-grade (G2) transitional cell carcinoma of the right ureter that was treated with a nephroureterocystectomy (partial cystectomy) five years previously. A biopsy showed many large Paget cells with abundant basophilic or amphophilic cytoplasm that contained prominent vesicular nuclei lying singly or in clusters in the epidermis of the glans penis (Figure 2). Immunohistochemical staining was positive for CK7, CK20 and GATA3, and negative for S-100, HMB-45 and GCDFP15. Neoplastic cells were positive for periodic acid-Schiff staining. We performed laboratory and radiologic examinations and excluded regional internal malignant disease. We diagnosed extramammary Paget disease secondary to transitional cell carcinoma of the right ureter.

Extramammary Paget disease is an intraepithelial adenocarcinoma with a predilection for apocrine gland-bearing areas, which can be classified into primary (intraepithelial adenocarcinoma arising within the epidermis) and secondary (intraepithelial spread of a visceral carcinoma) disease. The association of secondary extramammary Paget disease of the glans penis with urothelial carcinoma has been reported uncommonly. ${ }^{1}$

The clinical features of extramammary Paget disease are nonspecific; it usually presents with pruritic eczematous plaques. Therefore, a prompt biopsy is reasonable in a patient with eczematous rashes in apocrine gland-rich regions that are unresponsive to topical treatment. A panel of immunohistochemical staining that includes CK7, CK20, GCDFP-15, p63, GATA3 and UP III is useful to diagnose extramammary Paget disease and to distinguish primary from secondary disease. ${ }^{2}$

We referred our patient to the urology department for a resection of the glans penis. Four years after the diagnosis of extramammary Paget disease, the patient developed bladder metastases and died two years later.

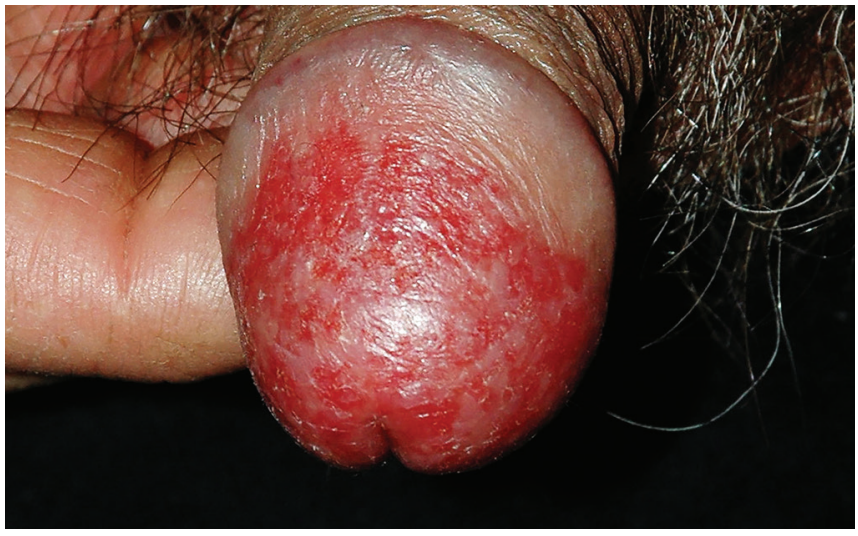

Figure 1: An irregular, circumscribed, infiltrated, erythematous plaque on the glans penis of a 63-year-old man with extramammary Paget disease.

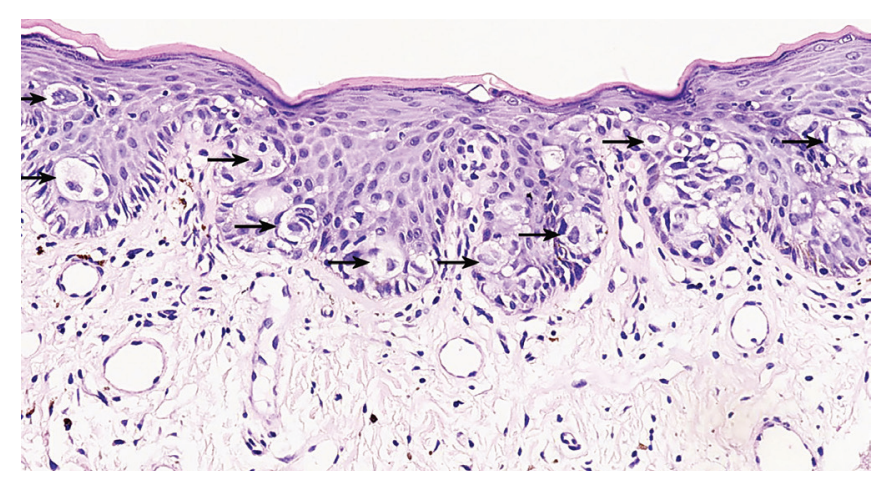

Figure 2: Numerous Paget cells lying singly or in clusters in the epidermis of the glans penis (arrows). Hematoxylin and eosin stain. Original magnification $\times 400$.

\section{References}

1. Martín-Fuentes A, Sánchez-Herreros C, Cuevas-Santos J, et al. Extramammary Paget disease secondary to a transitional cell carcinoma of the bladder. Indian J Dermatol Venereol Leprol 2015;81:318-20.

2. Morbeck D, Tregnago AC, Baiocchi G, et al. GATA3 expression in primary vulvar Paget disease: a potential pitfall leading to misdiagnosis of pagetoid urothelial intraepithelial neoplasia (PUIN). Histopathology 2017;70:435-41.

\section{Competing interests: None declared.}

This article has been peer reviewed.

The authors have obtained consent from the patient's son.
Affiliation: Department of Dermatology, Peking Union Medical College Hospital, Chinese Academy of Medical Sciences, Beijing, China

Correspondence to: Dong-Lai Ma, mdonglai@sohu.com 